\title{
Разогрев и релаксация энергии электронно-дырочного газа в треке первичного атома отдачи
}

\author{
(C) А.С. Пузанов ${ }^{1,2}$, С.В. Оболенский ${ }^{1,2}$, В.А. Козлов ${ }^{3}$ \\ ${ }^{1}$ Нижегородский государственный университет им. Н.И. Лобачевского, \\ 603950 Нижний Новгород, Россия \\ ${ }^{2}$ Филиал Российского федерального ядерного Всероссийского научно-исследовательского института \\ экспериментальной физики „Научно-исследовательский институт измерительных систем им. Ю.Е. Седакова“, \\ 603950 Нижний Новгород, Россия \\ ${ }^{3}$ Институт ффизики микроструктур Российской академии наук, \\ 603087 Нижний Новгород, Россия \\ E-mail: aspuzanov@inbox.ru
}

Поступила в Редакцию 15 апреля 2020 г.

В окончательной редакции 21 апреля 2020 г.

Принята к публикации 21 апреля 2020 г.

На основе алгоритма Монте-Карло разработан метод расчета энергетического спектра горячих неравновесных электронов и дырок в треке первичного атома отдачи при воздействии одиночных быстрых нейтронов. Проведены расчеты разогрева и последующей релаксации неравновесных носителей заряда в кремнии в треке заряженной частицы с начальными энергиями в диапазоне 50-200 кэВ. Получены характерные температуры электронной и дырочной плазмы, которые составили 5400 и $2700 \mathrm{~K}$ соответственно. Обсуждается влияние радиационно-индуцированного разогрева носителей заряда на сбоеустойчивость элементов статической памяти.

Ключевые слова: первичный атом отдачи, обратимый одиночный сбой, горячие носители.

DOI: $10.21883 /$ FTP.2020.08.49627.03

\section{1. Введение}

Основной тенденцией развития цифровой и аналоговой твердотельной электроники является уменьшение размеров полупроводниковых структур, что приводит к улучшению их характеристик: уменьшению энергии переключения цифровых элементов и уменьшению времени пролета электронами и дырками рабочей области аналоговых элементов. Это обусловливает рост числа элементов цифровых микросхем и увеличение предельных частот аналоговых устройств. При этом уменьшение размеров полупроводниковых элементов до размеров порядка длины релаксации энергии ( 30 нм для электронов в кремнии) приводит к усилению физических эффектов, связанных с горячими носителями заряда $[1,2]$.

Детальный расчет спектра возбуждений вторичных частиц возникает в ряде задач радиационной физики, например, неупругом торможении заряженных частиц высоких энергий и связанного с ним комплекса процессов трекообразования [3-5]; оптимизации материала детекторов излучений по критерию энергетического разрешения [6] и т.д. Основной акцент в этом случае делается на анализе движения первичной частицы, процессы релаксации энергии и переноса вторичных частиц не рассматриваются.

Другой группой задач, требующей расчета спектра горячих носителей заряда, является моделирование процесса ударной ионизации. Впервые функция распределения горячих электронов и дырок в кремнии была получена в аналитическом виде в работе [7], численно - в работе [8]. В работе [9] предложена единая феноменологическая модель, связывающая наблюдения ударной ионизации в сильных электрических полях с данными по средней энергии генерации электронно-дырочной пары при воздействии заряженных частиц высоких энергий или $\gamma$-квантов. В литературе представлены работы по моделированию методом Монте-Карло переноса электронов в объемном кремнии при воздействии мягкого рентгеновского излучения [10-12], а также расчету функции распределения горячих носителей заряда в кремнии при воздействии ультрафиолетового фемтосекундного лазера [13].

В работе [14] представлена вычислительная методика и результаты расчетов спектральных распределений возбуждений твердотельной плазмы кремниевой транзисторной структуры ,металл-диэлектрик-полупроводник“ при воздействии заряженных частиц космического пространства. Показано [15], что основная часть горячих электронов концентрируется в сравнительно малой области вблизи дна зоны проводимости и имеет резкий спад, начиная с энергии 3.8 эВ. Расчет проведен в приближении торможения за счет генерации плазмонов, что предполагает большие скорости и энергии движущихся заряженных частиц, нереализуемые у первичных атомов отдачи при воздействии нейтронов спектра деления.

Ранее в работе [16] при помощи локально-неравновесной диффузионно-дрейфовой модели [17] было проведено моделирование обратимых одиночных сбоев функционирования ячеек статической памяти с проектными нормами 100-500 нм при воздействии потока 
мгновенных нейтронов спектра деления и интерпретирована серия облучательных экспериментов микросхем статической памяти с проектными нормами 350 нм. При этом температура неравновесных носителей заряда в треке первичного атома отдачи предполагалась равной температуре кристаллической решетки [18], что оправдано для указанных выше топологических норм, но может оказаться недостаточным для анализа сбоеустойчивости цифровых микросхем с нанометровыми топологическими нормами.

В данной работе проведено моделирование методом Монте-Карло обратимых одиночных сбоев микросхем памяти с проектными нормами 28 нм, изготовленных по технологии „кремний-на-изоляторе“ [19], с учетом разогрева и последующей релаксации энергии неравновесных носителей заряда в треке первичного атома отдачи при воздействии нейтронов спектра деления.

\section{2. Математическая модель}

В соответствии с современными представлениями [3-5] быстрый тяжелый ион формирует конусообразный непрерывный или прерывистый трек, состоящий из ядра, которое окружено оболочкой. Область ядра полностью ионизована, концентрация неравновесных носителей заряда достигает значений $2 \cdot 10^{23} \mathrm{~cm}^{-3}$, характерный радиус ядра составляет $r_{c}=\frac{v}{\omega_{p l}}$, где $v-$ скорость иона, $\omega=\sqrt{\frac{4 \pi n e^{2}}{m}}-$ плазмонная частота, $e$ и $m$ - заряд и масса электрона, $n-$ концентрация валентных электронов среды. В области оболочки энерговыделение спадает пропорционально $r^{-2}$, характерный радиус оболочки равен максимальной длине пробега $\delta$-электронов.

Рассмотренная модель с некоторыми ограничениями применима к трекам, формируемым первичными атомами отдачи, при воздействии нейтронов спектра деления. Во-первых, средняя энергия мгновенных нейтронов спектра деления составляет около 2 МэВ, следовательно, средняя энергия первичных атомов отдачи кремния составляет 140 кэВ. Таким образом, величина передаваемой энергии от первичного атома отдачи валентным электронам полупроводника не превосходит энергии объемных плазмонов $\left(\hbar \omega_{p l}=16.7\right.$ эВ для кремния $)$. Это означает, что единственным каналом возникновения неравновесных электронно-дырочных пар в этих условиях является генерация валентных электронов в зону проводимости. Во-вторых, в рассматриваемом диапазоне энергий возможна ионизация только близлежащих атомов, поэтому радиус ядра составляет один моноатомный слой. Как будет показано далее, радиус оболочки в этих условиях определяется диффузионным растеканием и охлаждением горячих неравновесных носителей заряда из ядра трека. Доминирующим механизмом потери энергии первичного атома отдачи является упругое ядерное

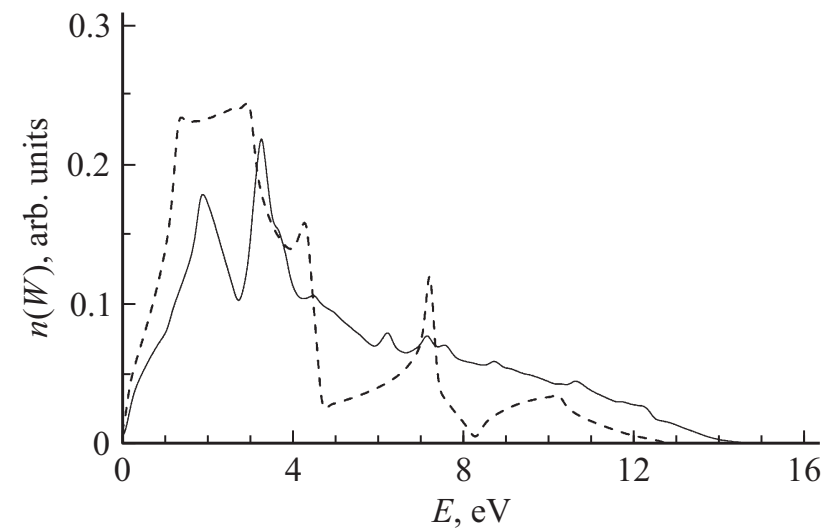

Рис. 1. Начальные распределения по энергии горячих неравновесных электронов (-) и дырок (---) в треке: $a-$ энергия первичного атома отдачи 205 кэВ, максимальная передаваемая энергия валентным электронам 16 эВ; $b$ - энергия первичного атома отдачи 154 кэВ, максимальная передаваемая энергия валентным электронам 12 эВ; $c$ - энергия первичного атома отдачи 103 кэВ, максимальная передаваемая энергия валентным электронам 8 эВ; $d-$ энергия первичного атома отдачи 51 кэВ, максимальная передаваемая энергия валентным электронам 4 эВ.

торможение, поэтому цилиндрическая структура трека справедлива только на участках между столкновениями.

\section{1. Энергетический спектр горячих неравновесных носителей заряда в ядре трека первичного атома отдачи}

В простейшем случае, функция плотности вероятности передачи энергии первичного атома отдачи валентным электронам может быть получена из дифференциального сечения рассеяния. Более точным представляется подход, основанный на диэлектрическом формализме. В этом случае распределение передаваемой энергии $p(\Delta E)$ может быть описано следующим выражением [6]:

$$
p(\Delta E) \propto \frac{1}{\frac{5 B\left(\Delta U_{\text {peak }}-1\right)^{3 / 2}\left(B \Delta U_{\text {peak }}+1\right)^{3 / 2}}{\sqrt{\frac{\Delta E}{E_{g}}}-1}+\left(B \frac{\Delta E}{E_{g}}+1\right)^{5 / 2}},
$$

где $\Delta E$ - величина передаваемой энергии, $E_{g}$ - ширина запрещенной зоны, $B=0.1$ и $\Delta U_{\text {peak }}=2.5$ для кремния - параметры модели.

После расчета передаваемой энергии необходимо определить распределение по энергии генерируемых электронов $E_{e}$ и дырок $E_{h}[6]$ :

$$
\begin{gathered}
p\left(E_{h}, E_{e} \mid \Delta E\right) \propto \rho_{v}\left(E_{h}\right) \rho_{c}\left(E_{e}\right), \\
E_{e}=\Delta E-E_{h}-E_{g},
\end{gathered}
$$

где $\rho_{v}\left(E_{h}\right.$ и $\rho_{c}\left(E_{e}\right)-$ функции плотности состояний дырок в валентной зоне и электронов в зоне проводимости. Для расчетов плотности состояний использовался метод эмпирического псевдопотенциала [20]. Начальные 
распределения по энергии горячих неравновесных носителей заряда в треке первичного атома отдачи кремния приведены на рис. 1.

\section{2. Релаксация энергии электронно-дырочного газа в треке первичного атома отдачи}

Для моделирования релаксации начального распределения по энергии горячих неравновесных носителей заряда в кремнии в треке первичного атома отдачи использовалась компьютерная программа для анализа переноса носителей заряда в субмикронных кремниевых структурах, ранее применявшаяся в работах [21-23]. Для расчета переходных ионизационных процессов в полевом транзисторе, изготовленном по технологии „кремний-на-изоляторе“, решалась самосогласованная задача движения макрочастиц в электрическом поле [24].

Для расчетов зонной структуры использовался метод эмпирического псевдопотенциала [20]. Так как рассматриваемая модель предназначена для анализа релаксации горячих электронов и дырок, то с целью экономии вычислительных ресурсов учитывались только механизмы рассеяния носителей заряда на оптических фононах, ударной ионизации и между собой. Частоты рассеяния на оптических фононах были взяты из работы [25], ударной ионизации - из работы [26], электрон-электронного рассеяния - из работы [3]. Отметим, что в разных работах [25,27-29] существует большой разброс частот рассеяния носителей заряда при больших энергиях носителей заряда. Поэтому основное внимание при подборе частот рассеяния уделялось соответствию значения насыщения дрейфовой скорости [28] и темпу ударной ионизации [30] в сильных электрических полях известным данным. Погрешность расчета составила не более $15 \%$.

Частоты рассеяния электронов при взаимодействии с оптическими фононами, ударной ионизации и между собой в зависимости от их энергии приведены на рис. 2. Несмотря на то что электрон-электронное рассеяние не

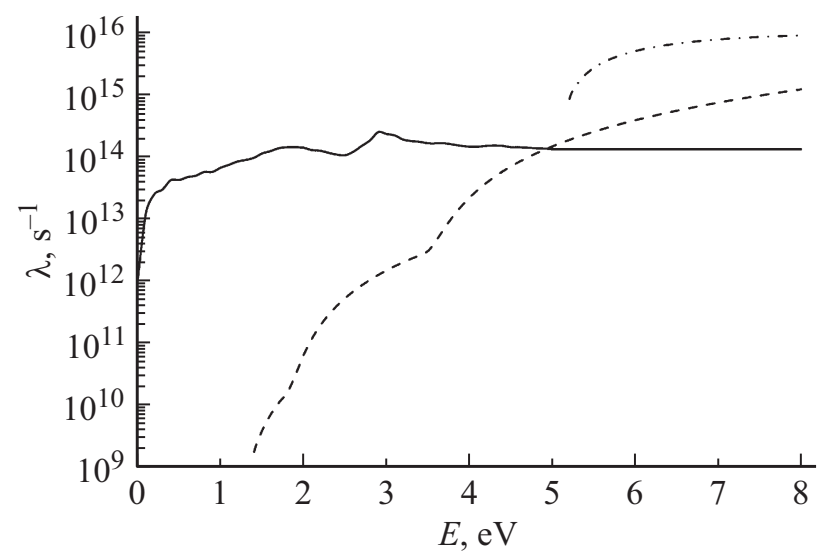

Рис. 2. Частоты рассеяния электронов в кремнии при взаимодействии с оптическими фононами $(-)$, ударной ионизации (---) и между собой $(-\cdot-\cdot-)$ в зависимости от их энергии. меняет среднюю энергию частиц, происходит перераспределение их импульсов, что влияет на общую динамику переноса носителей заряда и выноса энергии из трека. С уменьшением энергии электронов до 5 эВ влияние электрон-электронного взаимодействия уменьшается. Методика моделирования электрон-электронного рассеяния взята из работы [29].

\section{3. Результаты и обсуждение}

На первом этапе были получены начальные распределения по энергии горячих неравновесных носителей заряда в кремнии в треке первичного атома отдачи (рис. 1). Как следует из вышеприведенных формул, начальные распределения по энергии горячих неравновесных электронов и дырок определяются энергетической зависимостью плотности состояний носителей в валентной зоне и зоне проводимости, а также функцией распределения передаваемой энергии. Последнее обеспечивает монотонный спад функции распределения электронов при энергиях $>3.3$ эВ и дырок при энергиях $>10.3$ эВ.

Уменьшение энергии первичного атома отдачи приводит к снижению максимального значения передаваемой валентным электронам энергии. Это приводит к уменьшению граничной энергии носителей заряда в спектре и увеличению интенсивности распределения, так как неупругие потери энергии первичного атома отдачи в диапазоне энергий 50-200 кэВ меняются незначительно.

За время пролета первичного атома отдачи через область моделирования ( 0.1 пс) температуры электронов и дырок вдоль трека выравниваются и составляют $T_{e}=5400 \mathrm{~K}$ и $T_{h}=2700 \mathrm{~K}$ для максимальной передаваемой энергии 15.6 эВ, что соответствует энергии первичного атома отдачи кремния 200 кэВ. Уменьшение начальной энергии первичного атома отдачи до 40 кэВ не приводит к существенному изменению квазиустановившейся температуры за счет уменьшения характерных размеров оболочки трека. Отметим, что приведенные значения температур соответствуют экспериментально определенной электронной температуре в аморфном углероде для энергии налетающего иона 5 МэВ/а.е.м [4].

Дальнейшая релаксация энергии неравновесных носителей заряда происходит за счет взаимодействия с решеточной подсистемой и выноса энергии из трека за счет его диффузионного размытия.

Результаты расчетов переходных ионизационных процессов с учетом разогрева и последующей релаксации энергии горячих неравновесных носителей заряда в треке первичного атома отдачи с начальной энергией 200 кэВ в элементе микросхемы памяти с проектными нормами 28 нм, изготовленной по технологии „кремний-на-изоляторе“, приведены на рис. 3. Для сравнения также проведен расчет при помощи диффузионнодрейфовой модели [31], т.е. без учета радиационноиндуцированного разогрева носителей заряда. Рассматривался случай движения первичного атома отдачи 


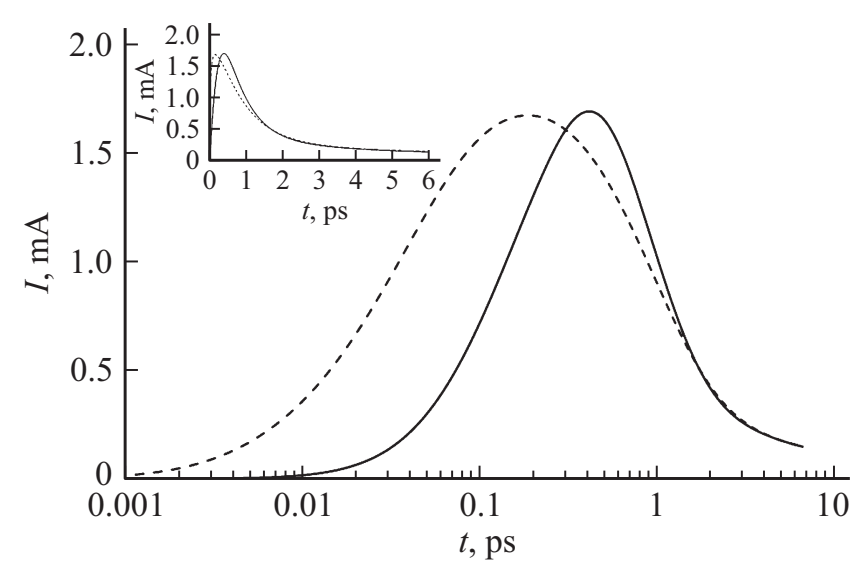

Рис. 3. Переходной ионизационный процесс при прохождении первичного атома отдачи с начальной энергией 200 кэВ через чувствительную область транзистора: $(-)$ - расчет методом Монте-Карло с учетом разогрева и релаксации энергии горячих неравновесных носителей заряда; (---) - расчет при помощи диффузионно-дрейфовой модели. На врезке зависимость переходного тока от времени приведена в линейном масштабе для демонстрации равенства величины собранного заряда.

вдоль поверхности микросхемы перпендикулярно линиям электрического тока в области стока транзистора, так как только такое направление обеспечивает достаточную длину трека и заряд для реализации обратимого одиночного сбоя [16]. В силу того что напряженность электрического поля в канале транзистора далека от пробойных значений, учет радиационно-индуцированного разогрева носителей не приводит к дополнительному их умножению. Поэтому максимальная амплитуда ионизационного тока $\left(I_{\max }=1.7 \mathrm{MA}\right)$ и величина собранного заряда $(Q=8.9$ фКл) для обеих моделей совпадают. Учет горячих носителей заряда в этих условиях приводит к увеличению интенсивности рассеяний на микроуровне и снижению подвижности на макроуровне. Таким образом, фронты отклика ионизационного тока затягиваются - носители медленнее „реагируют“ на изменение возмущающего воздействия.

Оценки стойкости к эффектам обратимых одиночных сбоев ячеек памяти в большинстве работ проводятся по величине критического заряда [32]. С позиции данного подхода учет радиационно-индуцированного разогрева неравновесных носителей заряда при напряжениях, далеких от пробивных значений, не влияет на оценку сбоеустойчивости микросхем. С другой стороны, работы ряда авторов [33] показали, что для стойкости к эффектам обратимых одиночных сбоев ячеек памяти необходимо учитывать не только величину критического заряда, но и форму возникающих всплесков тока. Следовательно, учет разогрева неравновесных носителей заряда в треке в общем случае влияет на оценку сбоеустойчивости микросхем.

Форма импульса тока определяется многими факторами: точкой возникновения первичного атома отдачи в структуре транзистора, начальной энергией и направлением развития каскада смещений. Значительное влияние оказывают реактивное сопротивление контактов и линий связи транзисторов в ячейке памяти. Расчеты показывают, что в первом приближении сбоеустойчивость субмикронной ячейки памяти определяется не только величиной собранного заряда, но и максимальной скоростью нарастания импульса тока, которая составляет $9 \cdot 10^{9} \mathrm{~A} / \mathrm{c}$ без учета и $4 \cdot 10^{9}$ с учетом радиационноиндуцированного разогрева неравновесных носителей заряда. Таким образом, в предельном случае расчетная оценка стойкости к эффектам обратимых одиночных сбоев субмикронных ячеек памяти различается более чем в 2 раза.

Данная ситуация объяснима с радиотехнической точки зрения. В том случае, если система обладает большой инерцией, т. е. длительность возбуждающего воздействия сушественно меньше времени переключения, то отклик определяется интегральной характеристикой воздействия и выполняется „дозовый“ критерий стойкости. Если система обладает малой инерцией, т.е. длительность возбуждающего воздействия существенно больше времени переключения, то отклик повторяет форму возбуждающего воздействия и выполняется „мощностной“ критерий стойкости. До недавнего времени время переключения ячеек памяти было существенно больше времени пролета заряженной частицы через чувствительный объем транзистора, что обеспечивало выполнение условий модели критического заряда. Уменьшение топологических норм позволило уменьшить время переключения и снизить инерционность цепей связи транзисторов в ячейке памяти. Это неизбежно приводит к необходимости детального расчета формы протекающего импульса тока, а также учета разогрева и релаксации энергии горячих неравновесных носителей заряда в треках заряженных частиц.

\section{4. Заключение}

Результаты проведенных расчетов показывают, что температура неравновесных носителей заряда в треке первичного атома отдачи существенно превосходит равновесное значение, задаваемое температурой кристаллической решетки и напряженностью электрического поля в канале транзистора. Учет радиационноиндуцированного разогрева электронно-дырочного газа приводит к изменению формы импульса тока, генерируемого заряженной частицей в субмикронных структурах. Интегральное значение заряда, собранного в канале транзистора, при этом не меняется. Форма импульсов тока влияет на переключение транзисторов в ячейках памяти, что приводит к необходимости детального расчета переходных ионизационных процессов с учетом разогрева и релаксации энергии горячих неравновесных носителей заряда в треках заряженных частиц для тео- 
ретического анализа сбоеустойчивости субмикронных микросхем.

\section{Финансирование работы}

Работа поддержана грантом Министерства науки и высшего образования РФ, полученным в рамках ФЦП „Исследования и разработки по приоритетным направлениям развития научно-технологического комплекса России на 2014-2020 годы“. Уникальный идентификатор проекта RFMEFI62020Х0003. Номер соглашения 075-15-2020-529.

\section{Конфликт интересов}

Авторы заявляют, что у них нет конфликта интересов.

\section{Список литературы}

[1] Ю. Пожела. Физика быстродействующих транзисторов (Вильнюс, Мокслас, 1989).

[2] М. Шур. Современные приборы на основе арсенида галлия (М., Мир, 1991).

[3] А.М. Митерев. Успехи физ. наук, 172 (10), 1131 (2002).

[4] Ф.Ф. Комаров. Успехи физ. наук, 173 (12), 1287 (2003).

[5] Ф.Ф. Комаров. Успехи физ. наук, 187 (5), 465 (2017).

[6] R.D. Narayan, R. Miranda, P. Rez. J. Appl. Phys., 111 (6), 064910 (2012)

[7] P.A. Wolff. Phys. Rev., 95 (6), 1415 (1954).

[8] E.O. Kane. Phys. Rev., 159 (3), 624 (1967).

[9] У. Шокли. Успехи физ. наук, 77 (1), 161 (1962).

[10] F.R. McFeely, E. Cartier, L.J. Terminello, A. Santoni, M.V. Fischetti. Phys. Rev. Lett., 65 (15), 1937 (1990).

[11] E. Cartier, F.R. McFeely. Phys. Rev. B., 44 (19), 10689 (1991).

[12] E.A. Eklund, P.D. Kirchner, D.K. Shuh, F.R. McFeely, E. Cartier. Phys. Rev. Lett., 68 (6), 831 (1992).

[13] N. Medvedev, B. Rethfeld. New J. Physics, 12, 073037 (2010).

[14] А.Г. Кадменский. Вопросы атомной науки и техники. Сер. Физика радиационного воздействия на радиоэлектронную аппаратуру, 1-2, 76 (1999).

[15] Г.М. Филиппов. ФТТ, 17 (7), 1920 (1977).

[16] А.С. Пузанов, М.М. Венедиктов, С.В. Оболенский, В.А. Козлов. ФТП, 53 (9), 1250 (2019).

[17] А.С. Пузанов, С.В. Оболенский, В.А. Козлов. ФТП, 52 (11), 1295 (2018).

[18] И.Ю. Забавичев, А.А. Потехин, А.С. Пузанов, С.В. Оболенский, В.А. Козлов. ФТП, 53 (9), 1279 (2019).

[19] M. Bo, G. Yong, S. Yi, Z. Hongwei, Z. Xing, L. Bo, L. Mengxin. 2018 IEEE 2nd Int. Conf. on Circuits, System and Simulation, p. 5.

[20] M.L. Cohen, T.K. Bergstresser. Phys. Rev., 141 (2), 789 (1966).

[21] А.С. Пузанов, С.В. Оболенский. Вопросы атомной науки и техники. Сер. Физика радиационного воздействия на радиоэлектронную аппаратуру, 3, 46 (2010).

[22] А.С. Пузанов, С.В. Оболенский, С.Г. Петров. Вопросы атомной науки и техники. Сер. Физика радиационного воздействия на радиоэлектронную аппаратуру, 2, 10 (2011).

[23] А.С. Пузанов, С.В. Оболенский. Микроэлектроника, 41 (4), 304 (2012).
[24] Р. Хокни, Дж. Иствуд. Численное моделирование методом частии (М., Мир, 1987) 640 с.

[25] J.Y. Tang, K. Hess. J. Appl. Phys., 54 (9), 5139 (1983).

[26] T. Kotani, M. van Schilfgaarde. Phys. Rev. B, 81 (12), 125201 (2010).

[27] C. Canali, C. Jacoboni, F. Nava, G. Ottaviani, A. AlberigiQuaranta. Phys. Rev. B., 12 (4), 2265 (1975).

[28] C. Jacoboni, L. Reggiani. Rev. Mod. Phys., 55 (3), 645 (1983).

[29] M. Fischetti, S. Laux. Phys. Rev. B., 38 (14), 9721 (1988).

[30] C.R. Crowell, S.M. Sze. Appl. Phys. Lett., 9 (6), 242 (1966).

[31] В.К. Киселев, С.В. Оболенский, А.С. Пузанов, А.В. Скупов. Журнал радиоэлектроники, 17 (2), 10 (2014).

[32] К.О. Петросянц, И.А. Харитонов, Е.В. Орехов, Л.М. Самбурский, А.П. Ятманов, А.В. Воеводин. Сб. $т р$. 5-й Всеросс. науч.-техн. конф. „Проблемы разработки перспективных микро- и наноэлектронных систем 2012“ (М., ФГБУ ИППМ РАН, 2012) с. 413.

[33] S. DasGupta. M.S. thesis, Dept. Electrion. Eng. and Comp. Science (Vanderbilt University, Dec. 2007) p. 116.

Редактор Г.А. Оганесян

\section{Heating and relaxation of the electron-hole gas energy in the track of the primary recoil atom}

\author{
A.S. Puzanov 1,2, S.V. Obolenskiy 1,2, V.A. Kozlov ${ }^{3}$ \\ ${ }^{1}$ Lobachevski State University of Nizhny Novgorod, \\ 603950 Nizhny Novgorod, Russia \\ ${ }^{2}$ Branch of Russian Federal Nuclear Center All-Russian \\ Research Institute of Experimental Physics „Sedakov \\ Scientific Research Institute of Measurement Systems“, \\ 603950 Nizhny Novgorod, Russia \\ ${ }^{3}$ Institute for Physics of Microstructure, \\ Russian Academy of Sciences, \\ 603087 Nizhny Novgorod, Russia
}

Abstract Based on the Monte Carlo algorithm, a method has been developed for calculating the energy spectrum of hot nonequilibrium electrons and holes in the track of the primary recoil atom when exposed to single fast neutrons. The calculations of the heating and subsequent relaxation of nonequilibrium charge carriers in silicon in the track of a charged particle with initial energies in the range of $50-200 \mathrm{keV}$ are carried out. The characteristic temperatures of the electron and hole plasma were obtained, which amounted to 5400 and $2700 \mathrm{~K}$, respectively. The effect of radiation-induced heating of charge carriers on the failure stability of static memory elements is discussed. 